\begin{tabular}{|c|l|}
\hline Title & In-bulk and surface structuring of sapphire by femtosecond pulses \\
\hline Author(s) & Juodkazis, Saulius; Nishimura, Koichi; Misawa, Hiroaki \\
\hline Citation & $\begin{array}{l}\text { A pplied Surface Science, 253(15), 6539-6544 } \\
\text { https://doi.org/10.1016/.apsusc.2007.01.097 }\end{array}$ \\
\hline Issue Date & 2007-05-30 \\
\hline Doc URL & http://hdl.handle.net/2115/28046 \\
\hline Type & article(author version) \\
\hline File Information & A SS253-15.pdf \\
\hline
\end{tabular}

Instructions for use 


\title{
In-bulk and surface structuring of sapphire by femtosecond pulses
}

\author{
Saulius Juodkazis, ${ }^{\mathrm{a}, \mathrm{b}}$ Koichi Nishimura, ${ }^{\mathrm{a}}$ Hiroaki Misawa ${ }^{\mathrm{a}, \mathrm{b}}$ \\ ${ }^{a}$ Research Institute for Electronic Science, Hokkaido University, North 21 - \\ West 10, CRIS Bldg., Kita-ku, Sapporo 001-0021, Japan \\ ${ }^{\mathrm{b}}$ Core Research for Evolution Science \& Technology (CREST), Japan Science \&6 \\ Technology Corporation (JST), Japan
}

\begin{abstract}
The actual space-time dependent intensity distribution of a tightly-focused (numerical aperture $N A=1.35$ ) Gaussian femtosecond pulse is modeled inside dielectric material. Such focusing is typically used for recording with sub-wavelength resolution inside dielectrics. The multi-pulse structuring inside the bulk and on the surface of sapphire are demonstrated. Formation of nano-cracks and nano-crystals is revealed inside the crystalline sapphire. Ripple formation on the surface is discussed in terms of the efficacy map calculated by theory given in J. E. Sipe et al., Phys. Rev. B, 27, 1141 (1983).
\end{abstract}

Key words: dielectric breakdown, femtosecond laser micofabrication, micro-explosion, sapphire

\section{Introduction}

Three-dimensional (3D) structuring of glasses, ceramics, crystals, and polymers by tightly focused femtosecond laser pulses is a promising technique for microfluidic, micro-optical and micro-mechanical applications. Tightly focused laser pulses can reach dielectric breakdown intensity (irradiance) without self-focusing when sub-1 ps pulses are used inside dielectric. Hence, a photo-structuring by 3D tightly focused laser pulses realizes the direct laser writing approach in a well controlled manner. By using objective lens of a high

Email addresses: Saulius@es.hokudai.ac.jp (Saulius Juodkazis,), Misawa@es.hokudai.ac.jp (Hiroaki Misawa). 

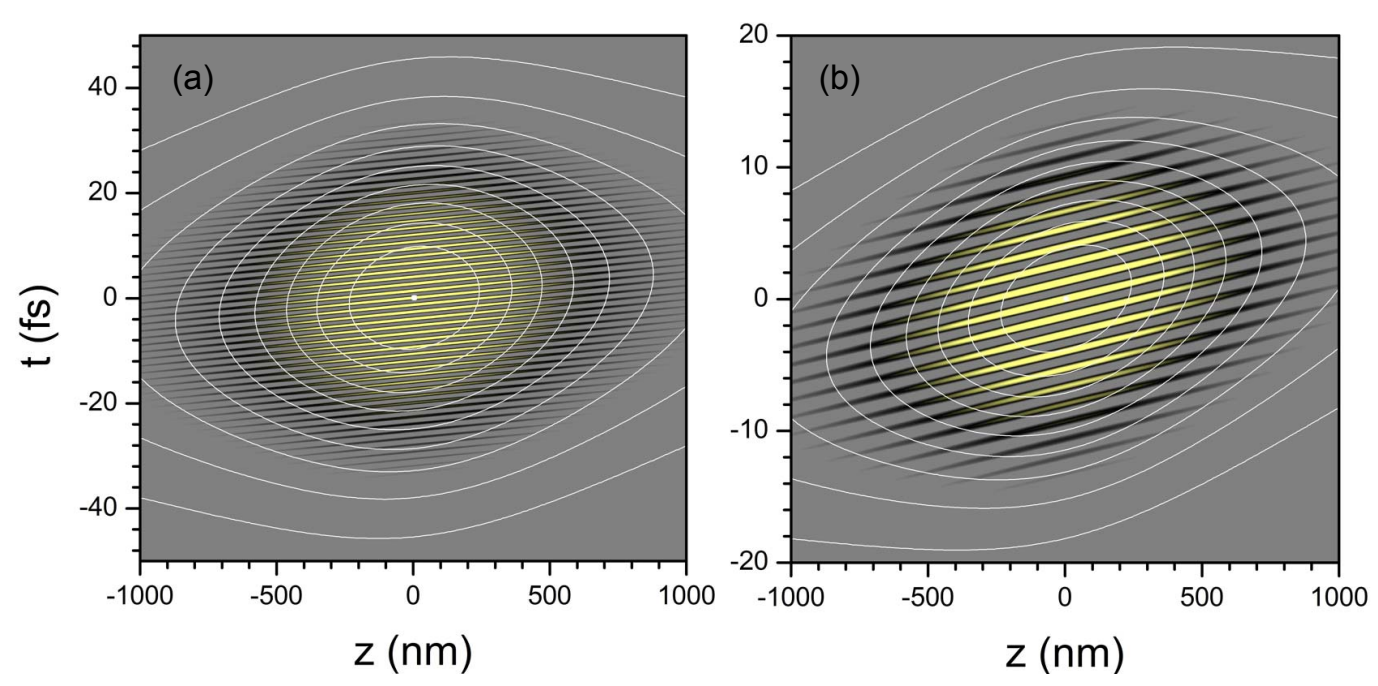

Fig. 1. (color online) The normalized intensity space-time map of $\left|\mathbf{E}\left(z, r, t^{\prime}\right)\right|^{2}$ at the focus: the intensity along z-axis of propagation at the focus for the $50 \mathrm{fs}$ (a) and $20 \mathrm{fs}$ (b) pulses at different time moments (vertical axis). Contour lines are drawn in a $10 \%$ steps over the envelope. The confocal parameter $2 z_{R}=1431 \mathrm{~nm}$.

numerical aperture $N A>1$ the focal spot size and, consequently, the photomodified region can be contained within a volume of sub-micrometer crosssections. The photomodifications can range from induced defects, color centers, polymerized voxels, and ultimately voids depending on material, focusing, and pulse energy. The mechanism of void formation [1] can be explained by nonlinear absorbtion and avalanche multiplication of electrons which effectively lead to ionization of the focal volume. The absorption in the plasma at focus is localized within a skin depth of tens-of-nanometers. This defines an ultimate localization of the energy delivery by a laser pulse. The high temperature and pressure buildup can be large enough to generate a shock wave (a strong micro-explosion). For example, a $100 \mathrm{~nJ}$ laser pulse can form a void under tight focusing conditions even in sapphire (Young modulus of $400 \mathrm{GPa}$ ). This opens new material processing routes for inert dielectrics [2]. The structural, physical, and chemical properties of nano-materials formed inside the shock-affected volume are expected to have novel properties not present in the bulk counterparts; e.g., the negative surface energy favors nano-crack and void growth in sapphire [3] or an annealing causes hardening of nano-phases of metal [4] (the opposite tendency to the macro-properties of the same metal).

Here, we discuss photo-structuring of sapphire by several femtosecond laser pulses under tight focusing. The focusing of an actual oscillating electric field is considered, since the focal length was comparable with the wavelength and, hence, a slowly varying envelope approximation (SVEA) is invalid for description of tight focusing. 


\section{Experimental}

Standard setup consisting of a laser providing amplified femtosecond pulses (Hurricane, Spectra Physics) and optical microscope was used for recording photo-modification inside sapphire under tight focusing $(N A=1.35)$ conditions. The central wavelength was $\lambda=800 \mathrm{~nm}$, pulse duration at focus $\tau_{p}=180 \mathrm{fs}$ (with chirp pre-compensation) at $1 \mathrm{kHz}$ repetition rate. The tilt of the pulses was minimized allowing for a good, plane wave-like, focusing. Single and multi-pulse irradiation was used. Morphology of photo-modified regions was inspected by scanning and transmission electron microscopies (SEM and TEM).

For ripples' recording a back-side irradiation by $100 \mathrm{~nJ}$ energy, $800 \mathrm{~nm}$ wavelength pulses focused on the surface of sapphire using the same $N A=1.35$ objective lens was used. Ablation lines were recorded at approximately 10$30 \mu \mathrm{m} / \mathrm{s}$ scan speed at a $1 \mathrm{kHz}$ repetition rate.

\section{Space-time dependent intensity at the focus}

We simulate the electrical field envelope at the focus by a Gaussian pulse in space and time, which in a cylindrical coordinate system is given as:

$$
E_{G}(z, r, t)=\frac{\left|\mathbf{E}_{\mathbf{0}}\right|}{1-i z \lambda /\left(w_{0}^{2} n \pi\right)} \exp \left(-\frac{r^{2} / w_{0}^{2}}{1-i z \lambda /\left(w_{0}^{2} n \pi\right)}\right) \exp \left(-2 \ln 2 \frac{t^{2}}{\tau_{p}^{2}}\right)
$$

where $i=\sqrt{-1}, z$ and $r$ are the axial and radial cylindrical coordinates, respectively, $\tau_{p}$ is pulse duration at FWHM, $w_{0}$ the waist, $\lambda$ is the vacuum wavelength, $\mathbf{E}_{\mathbf{0}}$ is the electric field amplitude, and $n$ is the refractive index. The oscillating E-field is then $E(z, r, t)=E_{G}(z, r, t) \times R e(\exp (i(\omega t-k z))$, here $k=2 \pi / \lambda$ is the wavevector. By substitution $t^{\prime}=t-z / c$, the obtained expression $E\left(z, r, t^{\prime}\right)$ can be used to reveal the space-time history of excitation. Figure 1 shows the axial cross-section of intensity at focus at different time moments, the space-time map. Here, the waist of the beam and the Rayleigh length are calculated as diffraction limit for a plane wave focusing according to $w_{0}=0.61 \lambda / N A$ (the numerical aperture $N A=1.35$ ) and $z_{R}=n \pi w_{0}^{2} / \lambda$, respectively. The central part of the focal region, approximately $\lambda / 2$ in length is enclosed inside the 0.9-contour line. When pulse duration is $20 \mathrm{fs}$, just a few optical oscillations reach the intensity $>0.9$. Also, every subsequent intensity peak enters the central focal part after the previous one has passed it (two intensity maxima occur per optical oscillation since $I \propto|\mathbf{E}|^{2}$ ). Obviously, this discussion does not take into account the ionization and effects of nonlinear 


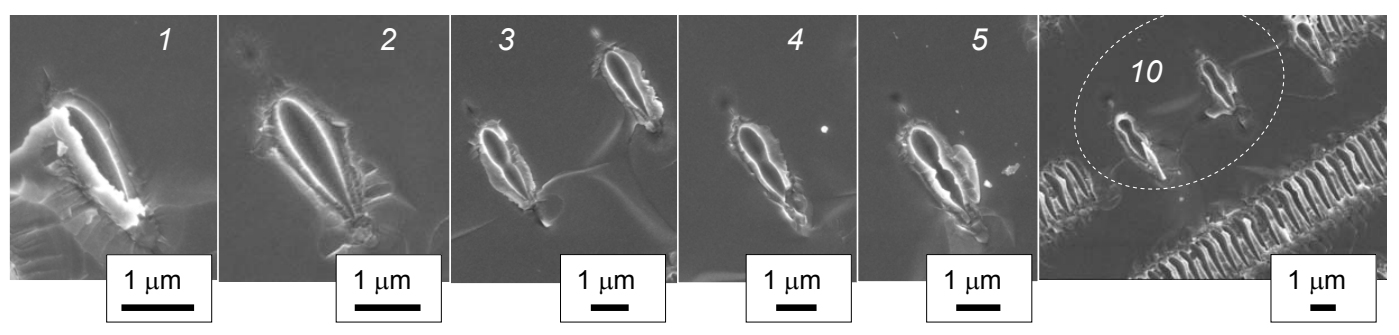

Fig. 2. SEM images of voids recorded by different number of $65 \mathrm{~nJ}$ pulses (shown by numbers) after wet etching in $5 \%$ aqueous solution of HF. The depth of recording was approximately $40 \mu \mathrm{m}$.

light propagation. The space-time map (Fig. 1) illustrates that a location and time dependent ionization is expected when the intensity reaches the threshold dielectric breakdown.

The dielectric breakdown starts by ionization at the focus (at the highest intensity location) and is localized to the typical skin depth of fully ionized region, approximately $50-100 \mathrm{~nm}[5]$ at irradiance $\sim 10-100 \mathrm{TW} / \mathrm{cm}^{2}$. A spherical shock-modified region would be expected from a micro-explosion scenario and was indeed observed for the voids recorded at small depth $(\sim 10 \mu \mathrm{m})$ where photo-modified region is close to spherical [1]. However, for a larger depth where spherical aberrations become considerable, a strongly elongated photomodification occurred [5]. It is noteworthy, that those regions were longer than would be expected from the focusing even taking into account spherical aberrations. The space-time dependent intensity should be considered in a modeling of filamentation in the case of the moving ionization.

\section{Multi-pulse damage of sapphire: bulk}

We found recently that multi-pulse exposure of sapphire changes the shockamorphised regions into poly-crystalline. This reduces chemical reactivity [2] of photo-modified regions and the channels recorded by continuous scanning become not wet etchable inside sapphire. Here, we have inspected by SEM voids recorded by exposure to increasing number of pulses without laser beam nor sample scanning. The results are summarized in Figure 2. It was possible to empty the multi-pulse irradiated sites by etching (Fig. 2); in contrary to the etching of channels formed at the same irradiation [2]. This is most probably due to the large nano-cracks density at the edge of photo-modified region and undamaged sapphire. The nano-cracks were preferentially in c-plane (Fig. 3). Such pattern of cracks should be present in the case of channel recording, however, the poly-crystalline phase formed in the central region of the channel hampered solubility and removal of the shock-affected sapphire from the channel; as a result no channel was formed [2]. Here, sample with the voids was 


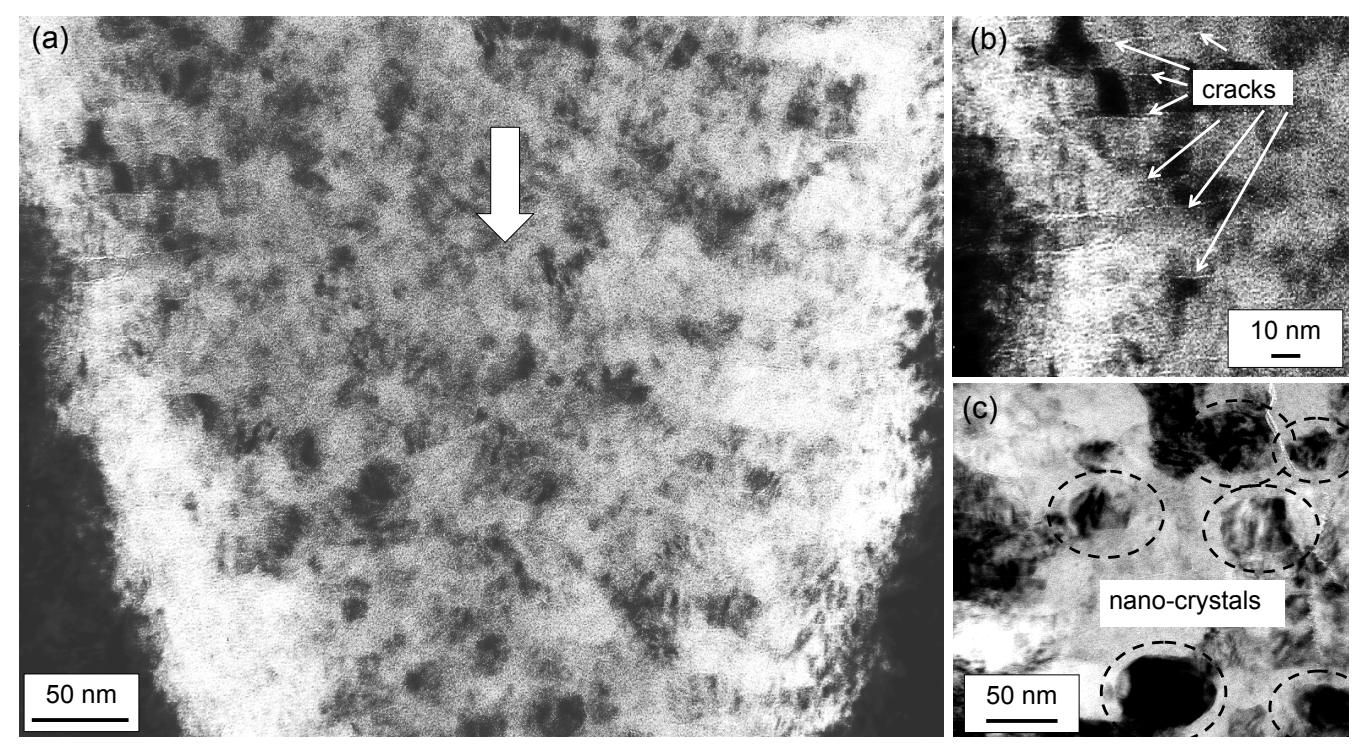

Fig. 3. (a) TEM image of a multi-pulse irradiated region in sapphire. Pulse energy $\sim 80$ nJ. Arrow marks direction of pulse propagation. (b-c) Closeup view of the edge and central part of image (a), respectively.

split in two halves and immersed into etching solution for better removal of material. This resulted in etching out of the optically damaged regions and revealed the extent of photo-modification by shock-wave propagation according to the model proposed recently [5].

It can be seen that the axial length of the shock-amorphised region (recognizable as a pit after etching) becomes saturated after several pulses. The pulse energy was large enough to create void at the center after the first pulse. The void and surrounding amorphous region become forward elongated after several pulses. This is a result of field enhancement at the front of the void for subsequent pulses as was simulated numerically [6].

\section{Multi-pulse damage of sapphire: surface}

Ripples were observed on the surface of laser irradiated materials in the first experiments on laser-matter interactions. This phenomenon is well understood as interaction of the irradiated and scattered waves at the boundary air-material [7]. Recently, a self-organization and a post-exposure surface reconstruction are shown to influence the observed ripple morphology [8]. Nevertheless, the irradiation geometry, wavelength, and, most importantly, the dielectric function of material are the main parameters determining the period and direction of ripples. In this section we discuss relation between the period of ripples and the electron concentration at which the ripples were formed based on theory presented in ref. [7] (details of simulations can be 


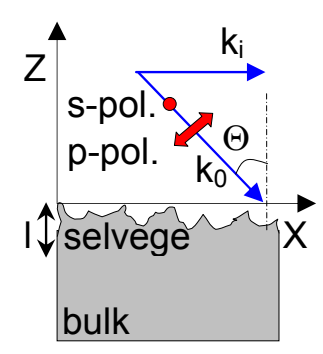

(a)

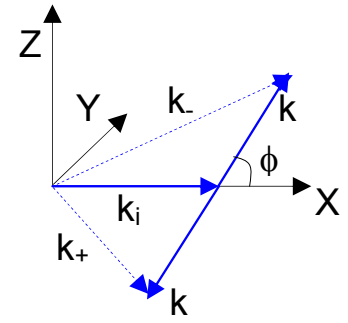

(b)

Fig. 4. (a) Geometry of numerical simulations: $k_{i}$ is the surface projection of the incident wavevector $k_{0}$, which is incident at angle $\theta, l$ is the depth of selvage region. (b) Definition of angle $\phi$ on the surface and wavevector of ripples, $k$ [7] $\left(k=2 \pi / \Lambda_{r}\right.$, where $\Lambda_{r}$ is the period of ripples); $k_{ \pm}$are auxiliary vectors used in theoretical model [7].

found elsewhere [9]).

Since the period of ripples is determined by the optical constants of material, it is expected to correlate with the electron concentration at the moment of ablation and ripple formation. We have tested this conjecture using sapphire and have compared the calculated electron density on the surface with that obtained in a 3D micro-structuring by shock-wave generation [1] at the same conditions of focusing and irradiance. Here, we do not consider a surface reconstruction by reflow of molten material due to large (among the largest) cohesion energy of Al-O, $E_{c o h} \simeq 4.5 \mathrm{eV} /$ bond [10] and its network-forming property (the coordination number of $\mathrm{Al}$ is 4 in oxide). The ripples can be considered as effectively "frozen" and their morphology been determined by light-matter interaction according to Sipe's theory [7] rather than by dynamics of molten material. The period of ripples observed on the surface of sapphire was approximately $\Lambda_{r} \simeq \lambda / 3 \simeq 267 \mathrm{~nm}$ (the period of ripples at the middle of ablation line in Fig. 5). It has been confirmed by number of experiments that the period of ripples at the normal incidence is $\Lambda_{r} \simeq \lambda$ and their wavevector is parallel to the polarization of incident light (the normal ripples), which had caused ripple formation [11]. This definition of ripples' period is valid for the frequency of light which is less than the plasma frequency (these constraints applies for our experiments).

\subsubsection{Optical properties of the breakdown plasma}

In electron-ion plasma according to the Drude's model the dielectric function 

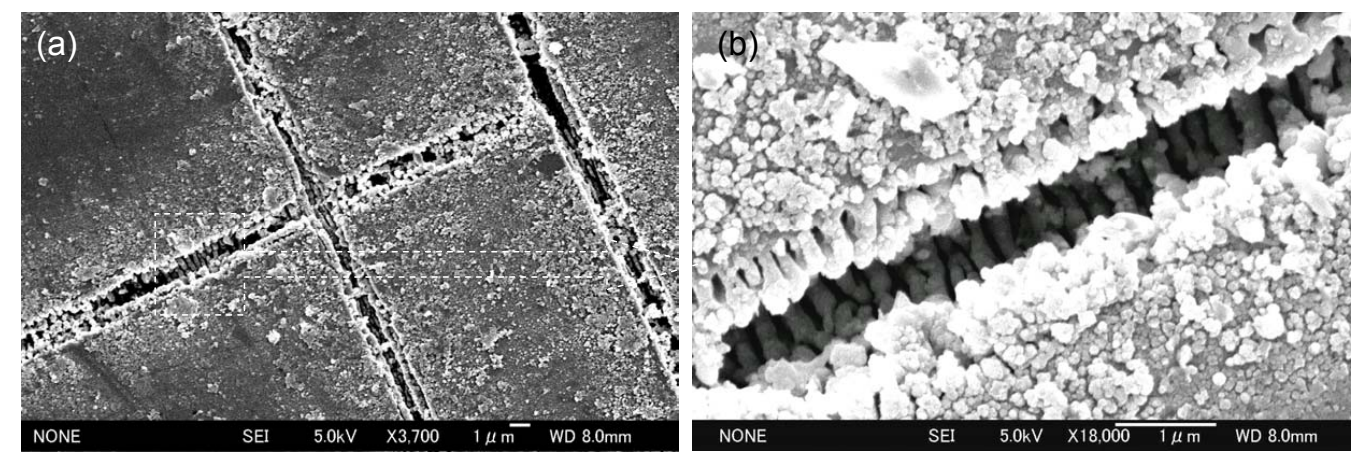

Fig. 5. SEM images of ablation lines on the surface of sapphire made by scanning $100 \mathrm{~nJ}$ pulses of $200 \mathrm{fs}$ duration at $800 \mathrm{~nm}$ wavelength. Focusing was carried out by an objective lens of numerical aperture $N A=1.35$; scanning speed approximately $10 \mu \mathrm{m} / \mathrm{s}$. Polarization was parallel to the ripple's wavevector $k$ (the normal ripples [11]).

$\varepsilon=\varepsilon_{1}+i \varepsilon_{2}$ is expressed as (e.g, see [11]):

$$
\begin{aligned}
& \varepsilon_{1}=1-\frac{\omega_{p}^{2}}{\omega^{2}+\nu_{e f f}^{2}}, \\
& \varepsilon_{2}=\frac{\omega_{p}^{2}}{\omega^{2}+\nu_{e f f}^{2}} \frac{\nu_{e f f}}{\omega},
\end{aligned}
$$

where the $\nu_{\text {eff }}$ is the effective electron - ion collision frequency, $\omega_{p}=\sqrt{\frac{N_{e} e^{2}}{\varepsilon_{0} m_{e}}}$ is the plasma frequency expressed via the electron density $N_{e}$, electron charge $e$, permittivity of free space $\varepsilon_{0}$, and optical mass of electron $m_{e}$ (usually, it is close to the actual mass of electron). By assuming $\nu_{\text {eff }} \sim \omega_{p}$, the real and imaginary parts of dielectric function and the refractive index $N \equiv \sqrt{\varepsilon}=n+i k$ are expressed as:

$$
\varepsilon_{1} \simeq \frac{\omega^{2}}{\omega_{p}^{2}} ; \varepsilon_{2} \simeq \frac{\omega_{p}}{\omega}\left(1+\frac{\omega^{2}}{\omega_{p}^{2}}\right)^{-1} ; n \simeq k=\sqrt{\frac{\varepsilon_{2}}{2}} .
$$

The absorption length is $l_{s}=c /(\omega k)$, where the $c$ is speed of light, $k$ is the extinction coefficient. For the normal incidence the absorbance, $A$, depends on the reflectivity, $R$, as $A=1-R \simeq 4 n /\left((n+1)^{2}+k^{2}\right)$.

The electron density at our irradiation conditions inside sapphire has been determined to be $N_{e} \simeq 5.3 \times 10^{23} \mathrm{~cm}^{3}$ [1] (at the same irradiance, wavelength, pulse duration, and focusing). This density corresponds to the multi-ionization of $\mathrm{Al}$ and $\mathrm{O}$ atoms. The atomic density of $\mathrm{Al}_{2} \mathrm{O}_{3}$ is $n_{a}=\rho N_{A} / M \simeq 2.35 \times$ $10^{22} \mathrm{~cm}^{-3}$, where the density $\rho=3.98 \mathrm{~g} / \mathrm{cm}^{3}$, the molar mass $M=101.96 \mathrm{~g}$, and $N_{A}$ is the Avogadro number. The ionization potentials of the "shallowest" electrons in $\mathrm{Al}$ are $5.98 \mathrm{eV}$ (one 3p-electron) and $24.8 \mathrm{eV}$ (two 3s-electrons) 
while for the O: $13.6 \mathrm{eV}$ (four 2p-electrons) and $28.5 \mathrm{eV}$ (two 2s-electrons). Shedding of several electrons from $\mathrm{Al}$ and $\mathrm{O}$ were required to reach electron density which was present at the ripple formation. Hence, $n \simeq k \simeq 2$ and the absorption coefficient $A \simeq 0.62$ at our experimental conditions with the E-field skin (absorption) depth $l_{s}=64 \mathrm{~nm}[1]$.

The electron energy density in the focal volume during the laser pulse can be estimated with all losses being neglected apart from the energy expenses for ionization [5]:

$$
E_{d e p}=\frac{2 A F}{l_{s}}-n_{a t} E_{i}
$$

where $E_{i}[\mathrm{~J}]$ accounts for the total losses of aluminium and oxygen ionization including a multiple ionization, and $n_{a t}$ is the atomic density of sapphire. The maximum pressure at the end of pulse can be estimated taking the whole deposited energy per the absorption volume. This pressure exceeds the Young modulus $E=400 \mathrm{GPa}$ ("cold" pressure) of sapphire and the shock wave is formed causing shock amorphisation [12]. The electron temperature, $T_{e}$, can be estimated from eq. 5. The maximum possible temperature of electrons when ionization losses $E_{i}$ and thermal conductivity are neglected can be found from energy conservation: $\partial c_{e} n_{e} T_{e} / \partial t=2 A F / l_{s}$, where $c_{e} \sim 3 / 2$ is the electron specific heat at full ionization and $n_{e} \sim 10^{23} \mathrm{~cm}^{-3}$ is the estimated electron density at the irradiance $\sim 10^{14} \mathrm{~W} / \mathrm{cm}^{3}$. One would find the electron temperature of $T_{e}=5.8 \mathrm{keV}$ at such conditions. The maximum thermodynamic temperature after energy equilibration between ions and electrons is approximately $T_{e} / 2$. It is noteworthy, that the emission of high temperature electrons can be used as a 3D localized spectrally broad emission source in photo-structuring [13].

\subsubsection{Efficacy of ripple formation}

The period of ripples depends mainly on the material's dielectric function at the irradiation wavelength, polarization, and incidence angle [7]. This can be presented collectively in an efficacy plot (a ripples' map) [9]. The maximum corresponds to the maximum intensity of the total field (incident and scattered). Interestingly, the other parameters, such as thickness of the selvege region where light scattering occurs (Fig. 4), properties of a liquid melt, its hydrodynamics, even the surface roughness, surrounding pressure and composition of atmosphere have only secondary effects on the ripples' period. This justifies the usage of the ripples' period as an imprint of the dielectric function at the moment of ripple formation and could be used to estimate the plasma density and temperature.

We calculate the efficacy plots for the ripples expected to be formed on a 

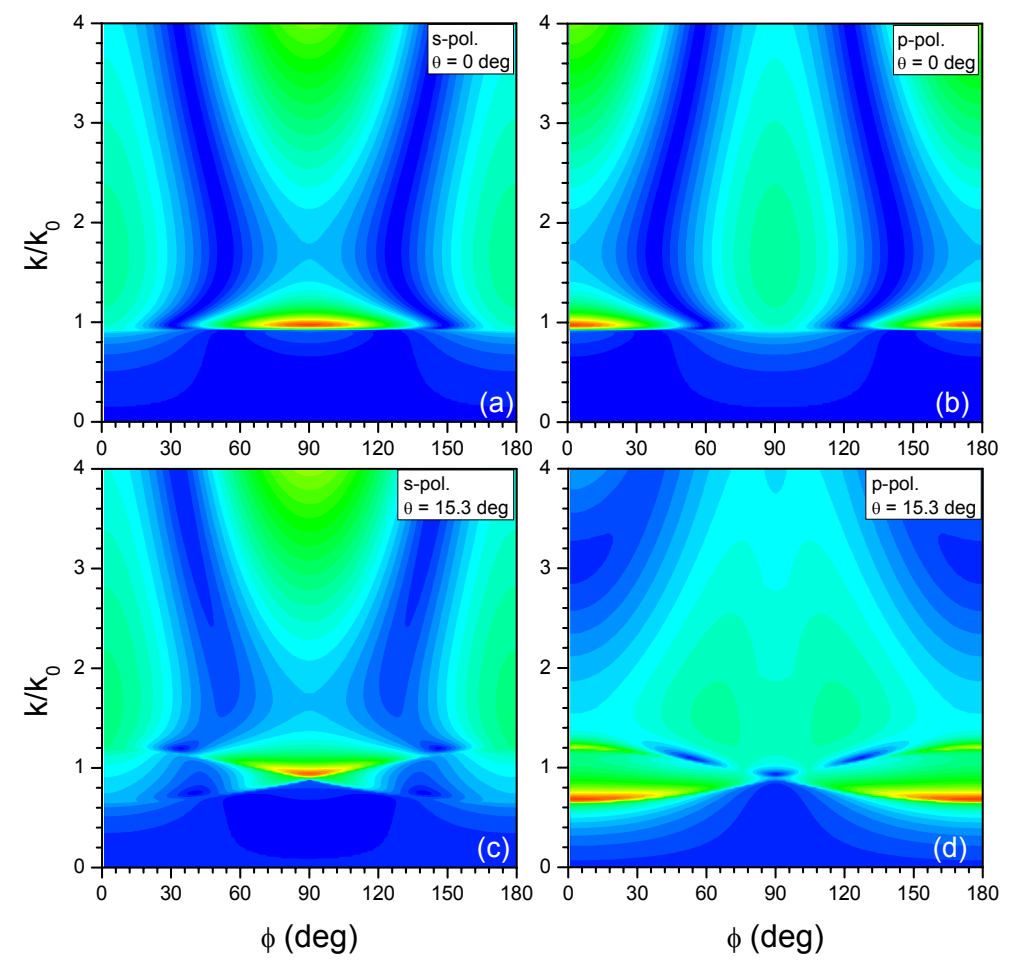

Fig. 6. (color online) Efficacy plots of ripples formed on the surface of material with refractive index $(2+2 i)$ (plasma) at the incidence angle $\theta=0$ (for polarizations $s$ (a) and $p(\mathrm{~b})$ ) and at $\theta=15.3$ degrees (c-d), respectively. The wavevector $k$ is normalized to the vacuum value $k_{0}$. The plots have intensity span $0-0.9(\mathrm{a}, \mathrm{b})$, while $0-0.8(\mathrm{c})$ and $0-1.3(\mathrm{~d})$.

surface of refractive index $(2+2 i)$ corresponding to the breakdown plasma at our experimental conditions. Figure 6 shows the efficacy factors for both s- and p-polarizations at two different incidence angles 0 and 15 degrees. The ablated surface morphology is expected to reflect the efficacy map, i.e., there should be ripples observed at certain angles $\phi$ on the surface and their wavevector corresponds to the maxima, minima, or kinks in the efficacy map. Since the tight focusing was used, it is not possible to define a single incident angle. The optical axis was perpendicular to the sample's surface, $\theta=0^{\circ}$, but the observed ripples did not follow the expected dependence shown in Fig. 6(a-b). Instead, the period of ripples is consistent with the efficacy minima position at the incidence angle $\theta=15^{\circ}$, which is the best fit angle (see, Fig. 6(d)) where $k / k_{0}=3$ and $\Lambda_{r} \simeq \lambda / 3\left(\right.$ at $\phi=0^{\circ}$ ) as observed in experiments. The $N A=1.35$ focussing corresponds to the incidence half-cone angle of $34^{\circ}$; hence, the most of pulse intensity is in the central part making the $\theta=$ $15^{\circ}$ angle a reasonable estimate for the effective ripple recording. Thus, there is a correlation between the dielectric function at the breakdown and the ripples period. The observed period of ripples corresponds to the minima of the efficacy factor at $\phi=0^{\circ}$ in Fig. 6(d). 


\section{Conclusions}

It is shown that the space-time dependent intensity at the focus is important when tight focusing is implemented. Such moving ionization can be an additional factor to the spherical aberration causing formation of the elongated damage sites in case of tightly focused fs-pulses [5]. The in-bulk laser structuring of sapphire at tight focusing conditions and high multi-pulse irradiance forms amorphous regions with complex nano-cracks and nano-crystals morphologies. The surface ablation of sapphire at similar conditions caused ablation and ripple formation. The period of ripples is shorter than the expected period at the right angle incidence and can be explained by the dielectric breakdown of the surface (the dielectric function of the breakdown plasma accounts for the observed period).

\section{References}

[1] S. Juodkazis, K. Nishimura, S. Tanaka, H. Misawa, E. E. Gamaly, B. LutherDavies, L. Hallo, P. Nicolai, and V. Tikhonchuk, "Laser-induced microexplosion confined in the bulk of a sapphire crystal: Evidence of multimegabar pressures," Phys. Rev. Lett. 96(16), p. 166101, 2006.

[2] S. Juodkazis, K. Nishimura, H. Misawa, T. Ebisui, R. Waki, S. Matsuo, and T. Okada, "Control over the state of crystallinity: Sapphire," Adv. Mat. 18(11), pp. 1361 - 1364 (DOI: 10.1002/adma.200501837), 2006.

[3] Z. Lodziana, N.-Y. Topsoe, and J. K. Norskov, "A negative surface energy for alumina," Nature Materials 3, pp. 289 - 293, 2004.

[4] X. Huang, N. Hansen, and N. Tsuji, "Hardening by annealing and softening by deformation in nanostructured metals," Science 312, pp. 249 - 251, 2006.

[5] E. E. Gamaly, S. Juodkazis, K. Nishimura, H. Misawa, B. Luther-Davies, L. Hallo, P. Nicolai, and V. Tikhonchuk, "Laser-matter interaction in a bulk of a transparent solid: confined micro-explosion and void formation," Phys. Rev. $B$ 73, p. 214101, 2006.

[6] T. Hashimoto, S. Juodkazis, and H. Misawa, "Void recording in silica," Appl. Phys. A 83(2), pp. 337 - 340 (DOI: 10.1007/s00339-006-3501-8), 2006.

[7] J. E. Sipe, J. F. Young, J. S. Preston, and H. M. van Driel, "Laser-induced periodic surface structure: I. Theory," Phys. Rev. B 27(2), pp. 1141 - 1154, 1983.

[8] G. D. W. Kautek, P. Rudolph and J. Krüger, "Physico-chemical aspects of femtosecond-pulse-laser-induced surface nanostructures," Appl. Phys. A 81(1), pp. $65-70,2005$. 
[9] S. Juodkazis, N. Kujime, H. Okuno, V. Mizeikis, S. Matsuo, and H. Misawa, "Towards nanostructuring of materials by ripples," in CRESTEQQNN'03 Joint Int. Workshop, Hyogo, Japan, pp. 117-121, July 21-23, 2003.

[10] K.-H. Sun, "Fundamental condition of glass formation," J. Am. Ceram. Soc. 30(9), pp. $277-281,1947$.

[11] D. Bäuerle, Laser processing and chemistry, Springer, Berlin, 3rd ed., 2000.

[12] S. Juodkazis, H. Misawa, E. E. Gamaly, B. Luther-Davies, A. Rode, L. Hallo, P. Nicolai, and V. Tikhonchuk, "Laser-matter interaction in a bulk of a transparent solid: confined micro-explosion," Journal of Physics: Conference Series (Proc. COLA-05), 2006 (in press).

[13] K. K. Seet, S. Juodkazis, V. Jarutis, and H. Misawa, "Feature-size reduction of photopolymerized structures by femtosecond optical curing of SU-8," Appl. Phys. Lett. 89, p. 024106, 2006. 